$\underline{\text { Review }}$

\title{
The evolution and role of interferon gamma release assays (IGRAs) in moderate and low income settings.
}

\author{
C.N. Ratnatunga \\ Sri Lankan Journal of Infectious Diseases 2014 Vol.4 (2):56-66 \\ DOI: http://dx.doi.org/10.4038/sljid.v4i2.7153
}

\begin{abstract}
The interferon gamma release assays have been extensively evaluated in the diagnosis of latent tuberculosis infection as well as in diagnosis of active pulmonary and extra-pulmonary tuberculosis since their introduction just over a decade ago. The results and recommendations published are confusing to say the least. The performance of these tests vary according to the population they are used in, including age, BCG vaccination status and immune status, the commercial test used, the TB burden of the country/ region and other factors intrinsic to the tests themselves. This review attempts to summarize the evolution of the IGRAs, particularly in the context of moderate and low income settings where a large question about their use still remains. Much work needs to be done before definitive recommendations about their role in the diagnostic algorithms of low and middle income countries can be made, but problems associated with the tests including cost and interpretation issues may limit the amount of work that can be invested productively in this evaluation.
\end{abstract}

Key words: tuberculosis, interferon gamma release assay, immunodiagnosis

\section{Introduction}

The interferon gamma release assays (IGRAs) were introduced just over a decade ago as a potential replacement for the century old tuberculin skin test (TST). The TST measures the type IV hypersensitivity reaction mounted in the skin in response to an injected cocktail of mycobacterial antigens (purified protein derivative or PPD). Numerous problems with the TST including lack of specificity for Mycobacterium tuberculosis (MTB) infection (as many non-tuberculous mycobacteria (NTM) also have these antigens), boosting of the response by BCG vaccination, necessity for a return visit by the patient, variable results depending on immune status of the patient, inter-reader variability in measurement and inability to differentiate between active and latent TB infection (LTBI) ${ }^{1}$ prompted the need for a new test which addressed at least some of these deficiencies.

The interferon gamma release assays are $\mathrm{T}$ cell function based assays that rely on immunological memory. $\mathrm{T}$ cells that have previously encountered Mycobacterium

Department of Microbiology, Faculty of Medicine, University of Peradeniya, Sri Lanka Address for correspondence: Dr. Champa Ratnatunga, Department of Microbiology, Faculty of Medicine, University of Peradeniya, Sri Lanka. Telephone No:+94 (0)776631106. Email - champa26@gmail.com

Received 16 July 2014 and revised version accepted 20 August 2014 
tuberculosis antigens (memory T cells), when exposed to the assay MTB antigens will become activated and produce interferon gamma (IFG) as a response. The IGRAs were developed using antigens that were more specific to Mycobacterium tuberculosis complex (MTBC) organisms to circumvent the problem of non specificity in the TST. The early secreted antigenic target 6 (ESAT-6) and culture filtrate protein 10 (CFP-10) are encoded by genes in the region of difference 1 (RD1) locus of the mycobacterial genome. Though they are not unique to the MTBC and are found in a few NTM including M. marinum, $M$. kansasii, $M$. szulgai and $M$ flavescens, they are not found in BCG/ $M$ bovis strains and are therefore theoretically not affected by BCG vaccination. ${ }^{1}$ In addition, this assay can be done and results obtained with a single visit of the patient, thereby obviating a second visit by the patient.

\section{The commercial assays}

Two companies currently manufacture and supply the interferon gamma assays as commercial blood test kits. The QuantiFERON-TB Gold (QFT-G) and QuantiFERON-TB Gold In-Tube (QFT-GIT) from Cellestis/ Qiagen, Carnegie, Australia and the T-SPOT.TB assay from Oxford Immunotec, Abingdon, UK. The QFT-GIT is a second generation test and is an ELISA (Enzyme Linked Immuno Sorbent Assay) that can be performed on whole blood, while the T-SPOT.TB assay is an ELISpot (Enzyme Linked Immuno Spot) assay that is performed using peripheral blood mononuclear cells (PBMC) separated from blood. The QFT uses the ESAT6 and CFP10 peptides as well as the TB7.7 peptide for T cell stimulation and the result is reported as a quantification of IFG in International Units (IU) per millilitre. The T-SPOT.TB assay uses only the ESAT6 and CFP10 antigens and the number of cells secreting IFG (spot forming cells) after antigenic stimulation is counted. Interpretation of both tests is based on positive and negative controls that are run with each sample and results are given as 'positive' 'negative' or 'indeterminate'.

Evaluating the assays: study types and contexts

Since their introduction in 2001, the performance of these tests has been evaluated in many different settings taking several variables into account. These include the local TB burden (high, moderate or low), BCG vaccination status of the population and the type of disease the test was used in (active pulmonary tuberculosis (PTB), extra-pulmonary tuberculosis (EPTB) or as a screening test for LBTI). As a gold standard method for diagnosis of LTBI is unavailable, active TB was often used as a surrogate outcome measure in many studies that evaluated sensitivity, while specificity was evaluated in low- burden low-risk populations. ${ }^{2-4}$

Sensitivity and specificity

Based on studies done in high, moderate and low burden settings between 2004 and 2008 using active TB as a surrogate outcome, the pooled sensitivity of the QFT-G was shown to be $78 \%$ (95\% CI 73-82\%), with point estimates ranging from 55\% to $88 \%$. The pooled sensitivity of the QFT-GIT was 70\% (95\% CI 63-78\%), with individual values ranging from $61 \%-93 \%{ }^{5-14}$ The pooled sensitivity of the T-SPOT.TB was estimated to be $90 \%$ (95\% CI $86-93 \%$ ) with individual values ranging from $83-100 \%$. The pooled sensitivity estimate for 
the TST was $77 \%$ (95\% CI 71-82\%) in these studies, with individual values ranging from $57 \%-100 \% .^{5-14}$ Of the studies that directly compared the T-SPOT.TB and the QFT-G or QFT-GIT, the majority showed that the T-SPOT.TB had a higher sensitivity. ${ }^{15}$

Specificity of the IGRAs were evaluated in both BCG vaccinated and non-vaccinated populations. These studies were done in low and moderate burden countries in apparently healthy adults who were assumed to have low risk of LTBI. The pooled specificity (all studies using both IGRAs) in BCG non-vaccinated populations was 99\% (98-100\%) with point estimates ranging from $95 \%$ to $100 \%$. The pooled specificity in BCG vaccinated populations was $96 \%$ (94\%-98\%), with point estimates ranging from $92 \%$ to $99 \%$. $^{14}$

Later analyses that compared moderate/ high and low burden settings showed that the QFT had a sensitivity of 69\% (95\%CI 64-73\%) in moderate/high burden settings while in low burden settings, the sensitivity was significantly higher at $83 \%$ (95\%CI 80-86\%). Sensitivity estimates were also prone to more variation in moderate/ high burden settings ${ }^{3}$.

Specificity estimates in moderate to high burden settings, including Zambia, South Africa, India, Tanzania, Turkey and China were markedly lower than in low burden settings with pooled estimates of 61\% (95\%CI $40-79 \%)$ for the T-SPOT.TB assay and 52\% (95\%CI 41$62 \%$ ) for the QFT assay being reported. ${ }^{16}$ These values however included both HIV infected and uninfected subjects. There was inadequate data to evaluate the pooled specificity of the IGRAs in HIV-uninfected persons. These estimates show that both sensitivity and specificity of the IGRAs were lower in high/ moderate burden settings. ${ }^{3,16}$

The specificity of the TST was shown to be good (97\%, 95\% CI 95-99\%) in BCG-non vaccinated populations. However this reduced to $59 \%$ (95\% CI 46-73\%) in BCG vaccinated populations. ${ }^{14}$ These values were calculated based on studies done in predominantly low burden settings in individuals at low risk of TB.

Based on results obtained from these studies as well as numerous other studies that evaluated the IGRAs in specific situations, the IGRAs were included in the diagnostic and screening algorithms of high-income countries including the USA ${ }^{17}$, Europe ${ }^{18}$ and Canada. ${ }^{19}$ They were recommended for use in conjunction with clinical history and risk assessment (including immune status and comorbidities of the patient) as well as other investigative tests results.

In contrast, in 2011 the WHO Strategic and Technical Advisory Group for Tuberculosis (STAG-TB) published recommendations that discouraged the use of IGRAs in low and middle income countries. ${ }^{20}$ The report recognised that IGRAs were being promoted and used, particularly in private sector hospitals and laboratories, for diagnosis of active TB. As high/ moderate burden settings are likely to have high LTBI rates in the population, the recommendations noted that the IGRAs were likely to have poor specificity as they cannot differentiate active from LTBI. Several IGRA studies $(n=22)$ done in low and middle income settings were evaluated and based on those results, the use of IGRAs and the TST in diagnosis of active PTB and EPTB in these settings was discouraged by the WHO. ${ }^{20}$ The use of IGRAs in place of the TST for LTBI in HIV infected patients was also not recommended. 
The general reason given for these recommendations was the lack of good quality evidence to support their use rather than findings of poor utility in these settings

Subsequent meta-analyses, done by Dai et al in 2012, compared 73 studies using both commercial IGRAs in China and other countries. They showed that the pooled sensitivity and specificity of the IGRAs was $85 \%$ (95\%CI $84-86 \%$ ) and $84 \%$ (95\%CI $83-85 \%$ ) respectively . The pooled estimates for the T-SPOT.TB test in the Chinese population gave a sensitivity of $88 \%$ (95\% CI 86-91) and a specificity of $89 \%$ (95\%CI $86-92 \%)$ while the estimates in nonChina studies were $90 \%$ (95\%CI 87-93\%) and 74\% (95\%CI 71-77\%) respectively. The sensitivity and specificity of the QFT overall were 78\% (95\%CI 75-80\%) and 81\% (95\%CI $80-83 \%)$. The authors concluded that while the IGRAs showed good sensitivity and specificity for diagnosis of active TB, their use should be decided based on local disease prevalence and other logistic considerations. ${ }^{4}$

The studies mentioned here comprise only a fraction of those done on IGRAs worldwide and highlight the variation in results that have been reported. Coming to a single uniform conclusion and developing practical guidelines has therefore been problematic resulting in different guidelines with differing recommendations. Ultimately the consensus appears to be that the tests must be validated for a given setting before their use. Similar variation in results and recommendations is seen in the use of IGRAs in the special circumstances mentioned below.

IGRA in immunocompromised patients

IGRAs were initially shown to have a higher sensitivity in immunocompromised populations leading to extensive investigation of their use in this subset. ${ }^{21}$ In immunocompromised patients with active PTB, studies have shown varying results with some showing that both the TST and the IGRAs have similar sensitivities in immunocompromised populations, while others show that both the IGRAs and TST have reduced sensitivity for active TB in compromised populations. ${ }^{18}$ Several studies showed that the T-SPOT.TB had a higher sensitivity in immunocompromised patients when compared to the QFT, but recommendations by the WHO remain that IGRAs should not be used for active TB diagnosis in the immunocompromised in low/middle income settings. ${ }^{20}$ These recommendations were supported by the findings of Metcalf et al (2011) who showed poor sensitivity and specificity of the IGRAs in immunocompromised patients in low/ middle income settings. ${ }^{16}$

\section{IGRA as a screening test}

The use of the IGRAs and the TST was compared in screening of healthy populations as well as in contact screening. Studies in low burden settings showed that the IGRAs had a better correlation to level of exposure than the TST while having the added advantage of not being affected by BCG vaccination. ${ }^{1,22-28}$ Studies in high burden countries showed the opposite with the TST correlating better with level of exposure than the IGRA. ${ }^{12,23,29,30}$ However, all these studies showed that both the TST and IGRA positivity correlated to some degree with level of exposure. 
In contact or other high risk category screening studies done in both high and low burden settings, most studies showed that the IGRAs gave lower positivity rates than the TST and when used as an alternative to the TST, or as a confirmatory test (2 step approach) would result in a reduction in LTBI diagnosis rates. ${ }^{31}$ However in high burden settings, studies have shown either no difference in both TST and IGRA positivity rates or higher positivity rates in IGRA ${ }^{32,33}$ In otherwise healthy populations (with no known exposure to TB) in both high and low burden settings, the IGRAs have been shown to have similar positivity rates to the TST. $^{34,35}$

Overall, current evidence points towards use of the IGRA alone or with the TST in low burden settings for screening at-risk patients but the use of IGRAs in high burden settings, particularly if LTBI is not treated, is debatable (and not recommended by the WHO) and would depend on assay cost, available laboratory infrastructure and objectives of the local TB screening programmes. Interpretation of results in high burden settings would also be problematic as there is no solid evidence base that points in one direction for use of IGRA in these settings.

The most recently published review by Pai et al, in 2014 was based on recent meta-analyses and extensively reviewed the available literature on IGRAs in all fields. ${ }^{14-16}$ This review stated that in low TB burden settings, the IGRAs have a specificity for LTBI (as assessed by surrogate markers) of $>95 \%$ which is not affected by BCG vaccination status. The TST also had a high specificity in this population. In BCG vaccinated populations, the specificity is lower and more variable in both tests. The sensitivity of the T-SPOT.TB test seems to be better than that of the QFT or the TST, but all have a sensitivity of over $70 \%$ in both populations. The IGRAs would be most useful for LTBI screening in populations where BCG vaccination is routinely done after infancy with multiple booster doses.

Other problems with the IGRAs

Test characteristics: repeated testing and variability in results

As the IGRAs are functional T cell assays, a large number of factors affect the final result of a given test. ${ }^{15}$ These include assay manufacturing and shipping, pre- analytical factors including time of phlebotomy, blood volume (not applicable to the T-SPOT.TB assay as the number of mononuclear cells is standardized), transportation temperature, incubation time and delay in starting incubation. Analytical factors including within-run, between-run, between-operator and between-laboratory imprecision and immunological / host factors such as boosting by previous PPD, other infections, immune status, age, and co-morbidities all have an effect on the test outcome. ${ }^{15}$

All factors that cause variability in IGRA results are not yet fully understood. Much of the research carried out to-date has been on the QFT test and sources of variation in the TSPOT.TB test are less well defined. As all these factors could affect the final outcome of a given test result, the reproducibility of results and the interpretation of a single positive or negative result remains problematic. 
Conversions and reversions occur with IGRA results. ${ }^{36}$ Follow up studies of both patients and contacts have shown mixed results. ${ }^{36}$ Some studies have shown that the IGRAs become negative during the course of treatment of active TB, while others have shown that IGRA conversions to positive occurred. ${ }^{36}$ Conversions and reversions also occur in contacts investigated in screening programmes as mentioned before. ${ }^{37}$ Conversions and reversions are categorized as stable and unstable, where stable conversions/ reversions are changes in results which persist for a long period of time, and unstable conversions/ reversions are changes that revert within a short period. ${ }^{15}$ As IGRAs are dynamic functional T cell assays, this phenomenon is expected. However interpretation of these results remains a problem.

\section{Indeterminate results}

Indeterminate results are seen in 5-40\% of the IGRAs. The most common reason for this is a failed positive control, which is associated with extremes of age and immune compromised status. ${ }^{2,16}$ Studies in immune compromised patients cite this as an advantage over the TST as a negative result can be distinguished from an indeterminate result due to anergy of T cells, whereas negativity of response and anergy cannot be differentiated in the TST. A significantly lower mitogen response has been shown in patients with active TB compared to controls in recent analysis and possible impairment of immune response has been put forward as a possible explanation for this. ${ }^{38}$ Laboratory factors such as delay in starting incubation have also been shown to affect the number of indeterminate results. ${ }^{39}$

\section{Other factors affecting IGRA result}

Multiple factors affect the IGRA result including malnutrition, levels of exposure to MTB antigens, transmission dynamics, and the $\mathrm{T}$ helper and $\mathrm{T}$ regulator cell cytokine profile of the host. ${ }^{3}$ It is also theorized that while early clearance of the organism from the lungs may cause a transient $\mathrm{T}$ cell response, LTBI may have no response because the organisms are dormant. $^{3}$

Though the IGRA is not affected by previous BCG vaccination, it has been shown to be affected by previous TST. Again, this finding is not consistent, with studies showing both the presence and absence of the boosting phenomenon. ${ }^{40-43}$

Recent evidence that IGRA response varies with age and the diabetic state is also emerging raising questions about the utility of these tests in elderly and diabetic populations. ${ }^{44-46}$ This presents important implications for countries where the population most likely to have TB infection are elderly or diabetic.

\section{$\underline{\text { Cost }}$}

Cost calculations have shown that the IGRAs are substantially more expensive to perform than the TST. Estimated cost for the T-SPOT.TB, including test kits, consumables, processing and phlebotomy is EUR 66.00 (GBP 55.00) while similar estimates for the QFT is EUR 54.00 (GBP 45). In contrast, the estimated cost for the TST, including consumables, administration and reading, is EUR 19.30 (GBP 16.14). ${ }^{18}$ However when other indirect costs were taken into account, including the reduced number of chest X-rays needed and reduced number of contacts and HCW who have to undergo preventive therapy when the IGRAs are used for LTBI screening, the IGRAs remain a cost effective method of diagnosis compared 
to the TST. ${ }^{18}$ Another study in a moderate incidence/ low income setting shows that the costs for the QFT was USD 18.60 while the cost of the TST was USD $3.70 .{ }^{47}$ The cost structure for the TST and IGRAs are different in that the bulk of the cost in the IGRAs is for consumables while most of the cost in TST is for time demands on participants. ${ }^{48}$

The local setting: where does Sri Lankan stand?

Sri Lanka is now classified as a moderate TB burden country. ${ }^{49}$ The TST is still used widely in contact screening and is also used in diagnosis of active PTB when smear microscopy is negative. IGRAs are available in the private sector hospitals and laboratories at great cost to patients. The test is often requested when tuberculosis (either PTB or EPTB) is suspected and other diagnostic tests have proved negative or inconclusive. There is currently no published data on the performance of the IGRAs in the local population in any form of the disease although preliminary studies have been conducted. Neither are there guidelines for the interpretation of single test results which is what is often done, as multiple testing is far too costly. The tests have not been validated for either diagnosis or screening in the Sri Lankan setting. No local data is available on how the test performs in specific groups such as immunocompromised patients (especially patients with renal failure and haematological malignancy) or children. With this lack of data, justification for doing this test, where a positive result does not necessarily indicate disease and a negative result does not exclude disease is indeed questionable.

\section{Conclusion}

The large amount of data available on the IGRAs has led to increased confusion rather than clarity. As seen in the above review of the literature, the IGRAs have been studied in many different contexts and results have been highly variable, with almost all findings being contradicted by findings in other studies. This state is reflected by the existing guidelines on IGRA use. A review of guidelines on IGRA use showed a large diversity of recommendations, and no consensus on their use in a given situation. ${ }^{50}$ This would perhaps indicate that until further evaluation of the IGRAs can be done, particularly in low and middle income settings, use of these assays should be limited or discouraged. Another approach would be to assess the situation and requirements in diagnostic and screening programmes in a local setting and then evaluate the IGRA for those specific needs. This approach would take into account local costs, population TB burden, laboratory facilities, age, BCG vaccination and immune status of the population studied, all of which have been shown to influence test utility and result. It would not be possible to generalize the results thus obtained to any other situation, but it would allow a more rational, need-oriented practice in a given setting.

Conflict of interest - The author declares no conflict of interest. 


\section{References}

1. Pai M, Riley LW, Colford JM. Interferon-gamma assays in the immunodiagnosis of tuberculosis: a systematic review. Lancet Infect Dis. 2004; 4(12):761-76. doi:10.1016/S1473-3099(04)01206-X.

2. Lalvani A, Pareek M. Interferon gamma release assays: principles and practice. Enferm Infecc Microbiol Clin. 2010; 28(4):245-52. doi:10.1016/j.eimc.2009.05.012.

3. Dheda K, van Zyl Smit R, Badri M, et al. T-cell interferon-gamma release assays for the rapid immunodiagnosis of tuberculosis: clinical utility in high-burden vs. lowburden settings. Curr Opin Pulm Med. 2009; 15(3):188-200. doi:10.1097/MCP.0b013e32832a0adc.

4. Dai Y, Feng Y, Xu R, et al. Evaluation of interferon-gamma release assays for the diagnosis of tuberculosis: an updated meta-analysis. Eur J Clin Microbiol Infect Dis. 2012; (31):3127-3137. doi:10.1007/s10096-012-1674-y.

5. Mori T, Sakatani M, Yamagishi F, et al. Specific detection of tuberculosis infection: an interferon-gamma-based assay using new antigens. Am J Respir Crit Care Med. 2004; 170(1):59-64. doi:10.1164/rccm.200402-179OC.

6. Ferrara G, Losi M, D'Amico R, et al. Use in routine clinical practice of two commercial blood tests for diagnosis of infection with Mycobacterium tuberculosis: a prospective study. Lancet. 2006; 367(9519):1328-34. doi:10.1016/S0140-6736(06)68579-6.

7. Ravn P, Munk ME, Andersen $\AA$ B, et al. Prospective evaluation of a whole-blood test using Mycobacterium tuberculosis -specific antigens ESAT-6 and CFP-10 for diagnosis of active tuberculosis. J Diagnostic Lab Immunol. 2005; 12(4):491-496. doi:10.1128/CDLI.12.4.491.

8. Lee JY, Choi HJ, Park I-N, et al. Comparison of two commercial interferon-gamma assays for diagnosing Mycobacterium tuberculosis infection. Eur Respir J. 2006; 28(1):24-30. doi:10.1183/09031936.06.00016906.

9. Tsiouris SJ, Coetzee D, Toro PL, et al. Sensitivity analysis and potential uses of a novel gamma interferon release assay for diagnosis of tuberculosis. J Clin Microbiol. 2006; 44(8):2844-50. doi:10.1128/JCM.02411-05.

10. Dewan PK, Grinsdale J, Kawamura LM. Low sensitivity of a whole-blood interferongamma release assay for detection of active tuberculosis. Clin Infect Dis. 2007; 44(1):69-73. doi:10.1086/509928.

11. Pai M, Menzies D. Interferon-gamma release assays: what is their role in the diagnosis of active tuberculosis? Clin Infect Dis. 2007; 44(1):74-7. doi:10.1086/509927.

12. Adetifa IMO, Lugos MD, Hammond A, et al. Comparison of two interferon gamma release assays in the diagnosis of Mycobacterium tuberculosis infection and disease in the Gambia. BMC Infect Dis. 2007; 7:122. doi:10.1186/1471-2334-7-122.

13. Barth RE, Mudrikova T, Hoepelman AIM. Interferon-gamma release assays (IGRAs) in high-endemic settings: could they play a role in optimizing global TB diagnostics? Evaluating the possibilities of using IGRAs to diagnose active TB in a rural African setting. Int J Infect Dis. 2008; 12(6):e1-6. doi:10.1016/j.ijid.2008.03.026.

14. Pai M, Zwerling A, Menzies D. Systematic Review: T-Cell - based assays for the diagnosis of latent tuberculosis Infection: An update. Ann Intern Med Rev. 2008; 149(3):177-184. doi:10.7326/0003-4819-149-3-200808050-00241 
15. Pai M, Denkinger CM, Kik S V, et al. Gamma interferon release assays for detection of Mycobacterium tuberculosis infection. Clin Microbiol Rev. 2014; 27(1):3-20. doi:10.1128/CMR.00034-13.

16. Metcalfe JZ, Everett CK, Steingart KR, et al. Interferon- $\gamma$ release assays for active pulmonary tuberculosis diagnosis in adults in low- and middle-income countries: systematic review and meta-analysis. J Infect Dis. 2011; 204(Suppl 4):S1120-9. doi:10.1093/infdis/jir410.

17. CDC. Updated guidelines for using interferon gamma release assays to detect Mycobacterium tuberculosis infection - United States. Atlanta; 2010. No doi.

18. ECDC. Use of interferon-gamma release assays in support of $T B$ diagnosis. Stockholm; 2011. No doi.

19. Menzies D, ed. Canadian Tuberculosis Standards. 7th Editio. Center for Communicable Disease and Infection Control, Public Health Agency of Canada; 2014. No doi.

20. WHO. Use of tuberculosis release assays ( IGRAs ) in low- and middle- income countries. Geneva, Switzerland: WHO/HTM/TB/2011.18; 2011. No doi

21. Aichelburg MC, Rieger A, Breitenecker F, et al. Detection and prediction of active tuberculosis disease by a whole-blood interferon-gamma release assay in HIV-1infected individuals. Clin Infect Dis. 2009; 48(7):954-62. doi:10.1086/597351.

22. Diel R, Ernst M, Döscher G, et al. Avoiding the effect of BCG vaccination in detecting Mycobacterium tuberculosis infection with a blood test. Eur Respir J. 2006; 28(1):1623. doi:10.1183/09031936.06.00107005.

23. Hill PC, Brookes RH, Fox A, et al. Large-scale evaluation of enzyme-linked immunospot assay and skin test for diagnosis of Mycobacterium tuberculosis infection against a gradient of exposure in The Gambia. Clin Infect Dis. 2004; 38(7):966-73. doi:10.1086/382362.

24. Arend SM, Thijsen SFT, Leyten EMS, et al. Comparison of two interferon-gamma assays and tuberculin skin test for tracing tuberculosis contacts. Am J Respir Crit Care Med. 2007; 175(6):618-27. doi:10.1164/rccm.200608-1099OC.

25. Ewer K, Deeks J, Alvarez L, et al. Comparison of T-cell-based assay with tuberculin skin test for diagnosis of Mycobacterium tuberculosis infection in a school tuberculosis outbreak. Lancet. 2003; 361(9364):1168-73. doi:10.1016/S0140-6736(03)12950-9.

26. Lalvani A, Pathan A A, Durkan H, et al. Enhanced contact tracing and spatial tracking of Mycobacterium tuberculosis infection by enumeration of antigen-specific T cells. Lancet. 2001; 357(9273):2017-21. doi:10.1016/S0140-6736(00)05115-1.

27. Shams H, Weis SE, Klucar P, et al. Enzyme-linked immunospot and tuberculin skin testing to detect latent tuberculosis infection. Am J Respir Crit Care Med. 2005; 172(9):1161-8. doi:10.1164/rccm.200505-748OC.

28. Zellweger J-P, Zellweger A, Ansermet S,et al. Contact tracing using a new T-cell-based test: better correlation with tuberculosis exposure than the tuberculin skin test. Int $J$ Tuberc Lung Dis. 2005; 9(11):1242-7. No doi.

29. Hill PC, Jeffries DJ, Brookes RH, et al. Using ELISPOT to expose false positive skin test conversion in tuberculosis contacts. PLoS One. 2007; 2(1):e183. doi:10.1371/journal.pone.0000183.

30. Kashyap RS, Nayak AR, Gaherwar HM, et al. Latent TB infection diagnosis in population exposed to TB subjects in close and poor ventilated high TB endemic zone in India. PLoS One. 2014; 9(3):e89524. doi:10.1371/journal.pone.0089524. 
31. Muñoz L, Santin M. Interferon- $\gamma$ release assays versus tuberculin skin test for targeting people for tuberculosis preventive treatment: An evidence-based review. $J$ Infect. 2013; 66(4):381-7. doi:10.1016/j.jinf.2012.12.005.

32. Person AK, Pettit AC, Sterling TR. Diagnosis and treatment of latent tuberculosis infection: an update. Curr Respir Care Rep. 2013; 2(4):199-207. doi:10.1007/s13665013-0064-y.

33. Shakak AO, Khalil EA, Musa AM, et al. Prevalence of latent tuberculosis infection in Sudan: a case-control study comparing interferon-gamma release assay and tuberculin skin test. BMC Public Health. 2013; 13(1):1128. doi:10.1186/1471-2458-13-1128.

34. Dagnew AF, Hussein J, Abebe M, et al. Diagnosis of latent tuberculosis infection in healthy young adults in a country with high tuberculosis burden and BCG vaccination at birth. BMC Res Notes. 2012; 5(1):1. doi:10.1186/1756-0500-5-415.

35. Hinks TSC, Varsani N, Godsiff DT, et al. High background rates of positive tuberculosis- specific interferon- $\gamma$ release assays in a low prevalence region of UK: a surveillance study. BMC Infect Dis. 2012; 12(1):1. doi:10.1186/1471-2334-12-339.

36. Mori T. Usefulness of interferon-gamma release assays for diagnosing TB infection and problems with these assays. J Infect Chemother. 2009; 15:143-155. doi:10.1007/s10156009-0686-8.

37. Pai M, Joshi R, Dogra S, Zwerling A. T-cell assay conversions and reversions among household contacts of tuberculosis patients in rural India. Int J Tuberc Lung Dis. 2009; 13(1):84-92. No doi.

38. Feng J, Huang $\mathrm{S}$, Lee $\mathrm{M}$, et al. Characteristics of IFN- $\gamma$ responses in IGRA among pulmonary TB suspects in a TB-endemic area. Diagn Microbiol Infect Dis. 2013; 77(1):46-52. doi:10.1016/j.diagmicrobio.2013.05.020.

39. Yun JW, Chung H, Koh W, et al. Significant reduction in rate of indeterminate results of the QuantiFERON-TB Gold In- Tube test by shortening incubation delay. J Clin Microbiol. 2014; 52(1):90. doi:10.1128/JCM.01547-13.

40. Van Zyl-Smit RN, Zwerling A, Dheda K, Pai M. Within-subject variability of interferon-gamma assay results for tuberculosis and boosting effect of tuberculin skin testing: a systematic review. PLoS One. 2009; 4(12):e8517. doi:10.1371/journal.pone.0008517.

41. Leyten EMS, Prins C, Bossink WJ, et al. Effect of tuberculin skin testing on a Mycobacterium tuberculosis-specific interferon-gamma assay. Eur Respir J. 2007; 29(6):1212-6. doi:10.1183/09031936.00117506.

42. Naseer A, Naqvi S, Kampmann B. Evidence for boosting Mycobacterium tuberculosisspecific IFN-gamma responses at 6 weeks following tuberculin skin testing. Eur Respir J. 2007; 29(6):1282-3. doi:10.1183/09031936.00017807.

43. Sauzullo I, Massetti AP, Mengoni F, et al. Influence of previous tuberculin skin test on serial IFN- $\gamma$ release assays. Tuberculosis (Edinb). 2011; 91(4):322-6. doi:10.1016/j.tube.2011.05.004.

44. Kamiya H, Ikushima S. Diagnostic performance of interferon-gamma release assays in elderly populations in comparison with younger populations. J Infect Chemother. 2013; 19:217-222. doi:10.1007/s10156-012-0480-x.

45. La Y, Nam Y, You E, et al. Factors influencing discordant results of the QuantiFERON-TB Gold In-tube test in patients with active TB. J Infect. 2013; 67(4):288-293. doi:10.1016/j.jinf.2013.06.005. 
46. Faurholt-jepsen D, Aabye MG, Jensen AV, et al. Diabetes is associated with lower tuberculosis antigen-specifi c interferon gamma release in Tanzanian tuberculosis patients and non-tuberculosis controls. Scandanavian J Infect Dis. 2014:March 2014. Early online:1-8. doi:10.3109/00365548.2014.885657.

47. Cardona $\mathrm{P}$, ed. Understanding tuberculosis - global experiences and innovative approaches. Rijeka: In Tech publishers, Croatia; 2012. No doi

48. Linertová R, Alvarez-León EE, García-Pérez L, Serrano-Aguilar P. Costs of QuantiFERON-TB Gold versus tuberculin skin test in Spanish healthcare workers. $J$ Hosp Infect. 2010; 75(1):52-5. doi:10.1016/j.jhin.2009.11.023.

49. WHO. Global Tuberculosis Report 2013. Geneva, Switzerland: WHO/HTM/TB/2013.11; 2013. No doi

50. Denkinger CM, Dheda K, Pai M. Guidelines on interferon- $\gamma$ release assays for tuberculosis infection: concordance, discordance or confusion? Clin Microbiol Infect. 2011; 17(6):806-14. doi:10.1111/j.1469-0691.2011.03555.x. 